Purdue University

Purdue e-Pubs

CTRC Research Publications

Cooling Technologies Research Center

2014

\title{
Droplet Evaporation on Heated Hydrophobic and Superhydrophobic Surfaces
}

S. Dash

Purdue University

S. V. Garimella

Purdue University, sureshg@purdue.edu

Follow this and additional works at: http://docs.lib.purdue.edu/coolingpubs

Dash, S. and Garimella, S. V., "Droplet Evaporation on Heated Hydrophobic and Superhydrophobic Surfaces" (2014). CTRC Research Publications. Paper 218.

http://dx.doi.org/http://dx.doi.org/10.1103/PhysRevE.89.042402

This document has been made available through Purdue e-Pubs, a service of the Purdue University Libraries. Please contact epubs@purdue.edu for additional information. 


\title{
Droplet evaporation on heated hydrophobic and superhydrophobic surfaces
}

\author{
Susmita Dash and Suresh V. Garimella* \\ Cooling Technologies Research Center, an NSF IUCRC School of Mechanical Engineering, and Birck Nanotechnology Center, \\ Purdue University, West Lafayette, Indiana 47907-2088, USA
}

(Received 25 January 2014; published 7 April 2014)

\begin{abstract}
The evaporation characteristics of sessile water droplets on smooth hydrophobic and structured superhydrophobic heated surfaces are experimentally investigated. Droplets placed on the hierarchical superhydrophobic surface subtend a very high contact angle $\left(\sim 160^{\circ}\right)$ and demonstrate low roll-off angle $\left(\sim 1^{\circ}\right)$, while the hydrophobic substrate supports corresponding values of $120^{\circ}$ and $\sim 10^{\circ}$. The substrates are heated to different constant temperatures in the range of $40-60{ }^{\circ} \mathrm{C}$, which causes the droplet to evaporate much faster than in the case of natural evaporation without heating. The geometric parameters of the droplet, such as contact angle, contact radius, and volume evolution over time, are experimentally tracked. The droplets are observed to evaporate primarily in a constant-contact-angle mode where the contact line slides along the surface. The measurements are compared with predictions from a model based on diffusion of vapor into the ambient that assumes isothermal conditions. This vapor-diffusion-only model captures the qualitative evaporation characteristics on both test substrates, but reasonable quantitative agreement is achieved only for the hydrophobic surface. The superhydrophobic surface demonstrates significant deviation between the measured evaporation rate and that obtained using the vapor-diffusion-only model, with the difference being amplified as the substrate temperature is increased. A simple model considering thermal diffusion through the droplet is used to highlight the important role of evaporative cooling at the droplet interface in determining the droplet evaporation characteristics on superhydrophobic surfaces.
\end{abstract}

DOI: 10.1103/PhysRevE.89.042402

PACS number(s): 68.08.Bc, 64.70.fm, 73.40.-c, 66.10.cd

\section{INTRODUCTION}

Droplet evaporation has important applications in inkjet printing [1], hot-spot cooling [2,3], surface patterning [4], droplet-based microfluidics [5], painting [6,7], biosensing [8], and DNA mapping $[9,10]$. It is important for proper design to understand the evaporation characteristics of a droplet in terms of the evaporation rate, flow pattern inside the droplet, deposition pattern of suspended particulates, and variation of contact angle (CA) and contact radius with and without substrate heating. Droplet evaporation characteristics depend on surface wettability [11], contact angle hysteresis [12], and surface roughness [13]. Two modes of evaporation were identified by Picknett and Bexon [14] for a droplet resting on a smooth surface, namely, the constant-contact-angle (CCA) mode and the constant contact radius (CCR) mode. The rate of evaporation of a sessile droplet was reported to be dependent on the contact radius and contact angle of the droplet [14]. Evaporation was also observed to begin in a CCR mode until the droplet reached the receding contact angle, at which point it transitioned to a CCA mode [14].

The interplay between factors such as the coupling between interface temperature and saturated vapor concentration; conduction through the substrate, droplet, and gas phases; convection in the liquid and gas domains; and the spherical-cap shape of the droplet, all complicate the determination of an exact solution for droplet evaporation rate. Most models in the literature treat evaporation as being induced only by vapor diffusion under isothermal conditions, subject to several simplifications in terms of the evaporative flux [15,16]. Popov [7] reported an exact, closed-form expression to describe the rate

*sureshg@purdue.edu of droplet evaporation by vapor diffusion for the range of contact angles. In recent studies, the substrate conductivity has been identified as being important in determining the evaporation rate of pinned sessile droplets $[17,18]$. Dunn et al. [18] proposed a model that included the effect of substrate thermal conductivity for a pinned sessile droplet with very low contact angle. Although the model could predict evaporation rates of volatile droplets, it underpredicted the evaporation rate for a water droplet.

Most prior studies have focused on droplet evaporation in a CCR mode [3,6,7,19]. Deegan et al. [20] suggested that the deposition of suspended particles in a droplet by the "coffee-ring" effect is attributable to a pinned contact line during evaporation and a nonuniform evaporation flux on the droplet surface (concentrated near the contact line). In many applications including inkjet printing [1], spotting of biofluids [21], and surface coating [22], the highly inhomogeneous solute deposition resulting from capillary-induced flow [6] is undesirable.

One means of exploiting evaporation to achieve localized deposition of suspended particles at the center of the footprint area of a droplet is through the use of a superhydrophobic surface with low contact angle hysteresis so that the CCA mode of evaporation is sustained [23]. McHale et al. [24] and Dash et al. [25] reported droplet evaporation on a superhydrophobic surface to follow three distinct phases: a CCR mode, a CCA mode, and a mixed mode in which both the contact angle and contact radius change simultaneously. A high initial droplet contact angle on a surface was earlier reported to be the required criterion for droplet evaporation to occur in the CCA mode [26,27]; however, droplet evaporation on superhydrophobic lotus leaves and biomimetic polymer surfaces, in spite of experiencing high initial contact angles $\left(\sim 150^{\circ}\right)$, has been reported to follow the CCR mode $[28,29]$. 
The mode of droplet evaporation has been shown to depend on the contact angle hysteresis on the surface, rather than on the initial contact angle of the droplet [12]. The low contact angle hysteresis renders superhydrophobic surfaces, with a sliding contact line during evaporation that thus suppresses the coffee-ring effect, a viable option for localizing the deposition of particles inside a droplet.

A survey of the literature indicates that studies concerning the concentration of suspended particles by means of droplet evaporation on superhydrophobic surfaces rely on diffusiondriven evaporation from an unheated substrate [30,31]. This results in long evaporation times (on the order of thousands of seconds). Applications such as molecule detection in biosensors [30] require a detailed understanding of the droplet evaporation dynamics on superhydrophobic surfaces, viz., the rate of evaporation and transient variation of contact angle or radius. Detection time can be reduced significantly upon the application of external heat to the droplet, but requires characterization of the evaporation characteristics (e.g., evaporation rate and transient droplet geometry) under heated conditions.

The vapor-diffusion model proposed by Popov [7] has been shown to predict the evaporation dynamics of droplets on hydrophilic substrates [6], hydrophobic substrates with sliding [32,33] and pinned [34] contact lines, and superhydrophobic substrates with pinned contact lines [29] with fair accuracy when the substrates are not heated. Recently, Dash and Garimella [32] experimentally demonstrated that the droplet evaporation rate on an unheated superhydrophobic surface with a sliding contact line was overestimated by this vapor-diffusion model [7] by approximately $20 \%$. Pan et al. [35] evaluated droplet evaporation under the same conditions using a comprehensive numerical model, and explained the discrepancy between earlier experimental results [32] and the vapor-diffusion-only model [7] in terms of the contributions of evaporative cooling and gas-phase convection effects which are not included in the model. Carle et al. [36] attributed the underestimation of evaporation rate by the vapor-diffusion model, in the case of hydrophilic substrates under heated conditions, to omission of the buoyancy-driven natural convection in the gas phase. Recently, Sobac and Brutin [34] discussed the influence of substrate temperature and substrate thermal properties on the evaporation behavior of droplets on hydrophilic and hydrophobic substrates. There has been limited investigation of the effect of substrate heating on the evaporation characteristics of droplets with a sliding contact line on hydrophobic and superhydrophobic substrates in the literature [29,32,34].

The present work investigates the evaporation characteristics of droplets when placed on heated hydrophobic and superhydrophobic surfaces with low contact angle hysteresis. The experimental results show that the vapor-diffusion-only model predicts the total time of droplet evaporation on a hydrophobic surface with reasonable accuracy. In the case of a superhydrophobic surface, however, the vapor-diffusion model overestimates the rate of evaporation significantly. A simple analytical model that includes the effects of thermal conduction through the liquid droplet in combination with vapor diffusion is presented to explain the shortcomings of the vapor-diffusion model in predicting the experimental results.

\section{VAPOR-DIFFUSION-ONLY EVAPORATION MODEL}

When no external heat is applied to the substrate, droplet evaporation is driven by the concentration gradient of water vapor between the droplet surface and the ambient. Diffusion of vapor through the atmosphere is the rate-limiting step; the time scale for diffusion is on the order of $R_{i}^{2} / D \sim 0.04 \mathrm{~s}$, where $D$ is the coefficient of vapor diffusion and $R_{i}$ is the initial radius of the droplet, on the order of millimeters [7]. The total time for complete evaporation of a droplet is significantly reduced when the surface is heated. However, even at a surface temperature of $60^{\circ} \mathrm{C}$, for a droplet of volume $3 \mu \mathrm{L}$, the ratio of diffusion time $\left(t_{D}\right)$ to evaporation time $\left(t_{F}\right)$ is $t_{D} / t_{F} \sim 0.0002$ (using experimental $t_{F}$ ), implying that the vapor concentration around the droplet may be assumed to be quasisteady [7]. Girard et al. [37] extended the vapor-diffusion model [7] by allowing the droplet and substrate to be at an elevated temperature with respect to the ambient (but the droplet still was at the same temperature as the substrate), and determined the evaporation rate of droplets on a hydrophilic heated substrate.

The vapor-diffusion model for evaporation [7], which is the exact solution to the Laplace equation in toroidal coordinates and accounts for the nonuniform vapor concentration around the droplet, can be extended to describe the evaporation flux $J(r)$ on the surface of the droplet on a heated substrate as $[34,37]$

$$
\begin{aligned}
J(r)= & \frac{D\left[c_{s}\left(T_{s}\right)-H c_{s}\left(T_{a}\right)\right]}{R_{c}} j(\theta), \\
j(\theta)= & {\left[\frac{1}{2} \sin \theta+\sqrt{2}(\cosh \alpha+\cos \theta)^{3 / 2}\right.} \\
& \times \int_{0}^{\infty} \frac{\cosh \theta \tau}{\cosh \pi \tau} \tan [(\pi-\theta) \tau] \\
& \left.\times P_{-(1 / 2)+i \tau}(\cosh \alpha) \tau d \tau\right],
\end{aligned}
$$

in which $T_{s}$ is the substrate temperature, $T_{a}$ the ambient temperature, $H$ the far-field relative humidity, $c_{s}$ the saturated vapor concentration on the droplet surface, $R_{c}$ the contact radius of the droplet, $\theta$ the contact angle of the droplet, and $r$ the radial coordinate at the base of the droplet such that $r=R_{c}$ at the contact line. The toroidal coordinates $\alpha$ and $\beta$ are related to the height $h$, contact radius $R_{c}$, and contact angle $\theta$ of the droplet as

$$
\cosh \alpha=\frac{\sin \theta}{\left(\frac{h}{R_{c}}\right)}-\cos \theta .
$$

Subsequently, for any contact angle, the rate of mass loss can be written as

$$
\begin{aligned}
& \frac{d M}{d t}=\rho_{L} \frac{d V}{d t}=-\pi R_{C} D\left[c_{s}\left(T_{s}\right)-H c_{s}\left(T_{a}\right)\right] f(\theta) ; \\
& f(\theta)=\frac{\sin \theta}{1+\cos \theta}+4 \int_{0}^{\infty} \frac{1+\cosh 2 \theta \tau}{\sinh 2 \pi \tau} \tanh [(\pi-\theta) \tau] d \tau,
\end{aligned}
$$

where $M$ is the droplet mass, $\rho_{L}$ the liquid density, $V$ the droplet volume, and $f(\theta)$ the functional variation of contact angle evaluated using a numerical integration scheme in 
MATLAB [38]. The vapor-diffusion model assumes the droplet to be maintained at the same temperature as the substrate. The properties of the droplet, viz., $\rho_{L}$ and $D$, are evaluated at the substrate temperature.

For droplet evaporation in the constant-contact-angle mode ( $\theta$ constant), with a spherical-cap assumption for droplet shape, the transient volume $V$ is obtained by integration of Eq. (3) and is given by

$$
\begin{aligned}
V^{2 / 3}= & V_{i}^{2 / 3}-\frac{2 \pi D\left[c_{s}\left(T_{s}\right)-H c_{s}\left(T_{a}\right)\right]}{3 \rho_{L}}\left(\frac{3}{\pi}\right)^{1 / 3} \\
& \times[g(\theta)]^{1 / 3} f(\theta) t \\
g(\theta)= & \frac{\sin ^{3} \theta}{(1-\cos \theta)^{2}(2+\cos \theta)}
\end{aligned}
$$

in which $V_{i}$ is the initial volume of the droplet. For constantcontact-angle evaporation, the time taken for complete evaporation $t_{\text {tot }}$ is given by

$$
t_{\mathrm{tot}}=\frac{\rho_{L}}{2 D\left[c_{s}\left(T_{s}\right)-H c_{s}\left(T_{a}\right)\right]}\left(\frac{3 V_{i}}{\pi}\right)^{2 / 3} \frac{1}{[g(\theta)]^{1 / 3} f(\theta)} .
$$

\section{METHODOLOGY AND EXPERIMENTAL SETUP}

The experiments are carried out on two substrates: a smooth hydrophobic surface and a hierarchical superhydrophobic surface. The hydrophobic surface is a silicon prime wafer spin coated with $0.2 \%$ solution of Teflon-AF 1600 (DuPont, Wilmington, DE) in FC-77 (3M, St. Paul, MN) to impart hydrophobicity. The hierarchical superhydrophobic surface is fabricated using the same methodology and has the same geometrical parameters as described in Dash and Garimella [32].

The experimental setup is shown in Fig. 1. The hot stage for the droplet evaporation experiments consists of a copper block that is electrically heated on its underside by a 10 W polyimide film heater (Minco); the sides of the block are insulated with fused ceramic foam. A PID controller (TOT-1200, Temp-o-Trol) is used to control the power input to the film heater based on the measured temperature close to the substrate. The test substrate is attached to the top of the copper block with a uniform layer of thermally conductive silicone paste (Omegatherm 201, Omega). The temperatures at four locations along the central axis of the copper block are continually measured during a test using

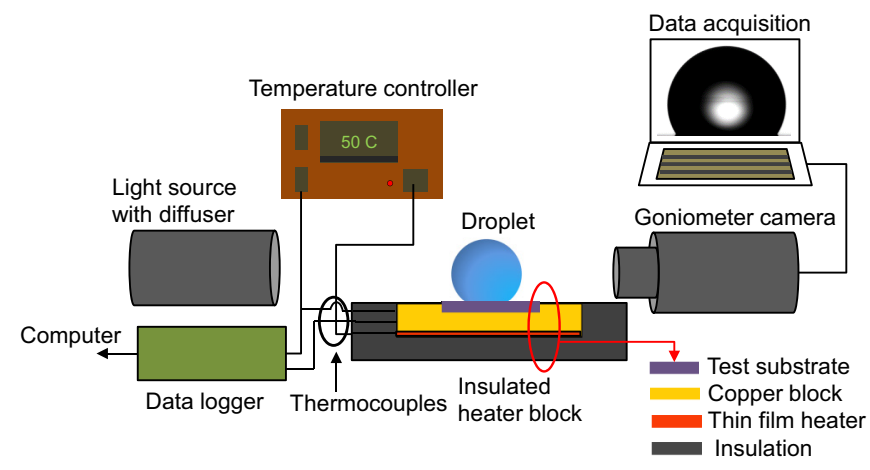

FIG. 1. (Color online) Schematic diagram of the experimental setup. a data acquisition system (34970A, Agilent Technologies). The temperature variation across the thickness of the copper block remained below the uncertainty of the thermocouple measurements. Therefore, the heater block can be treated as being isothermal, and the temperature nearest the sample substrate is quoted as the sample temperature for all the experiments. Evaporation experiments are carried out at three different substrate temperatures: $40 \pm 0.5^{\circ} \mathrm{C}, 50 \pm 0.5^{\circ} \mathrm{C}$, and $60 \pm 0.5^{\circ} \mathrm{C}$.

Deionized water droplets of initial volume $3 \mu \mathrm{L} \pm 0.1 \mu \mathrm{L}$ are used in the experiments, and have initial radii of $0.95 \mathrm{~mm}$ on the hydrophobic surface and $0.90 \mathrm{~mm}$ on the superhydrophobic surface. The characteristic length of the droplet (its radius) is smaller than the capillary length scale $(\gamma / \rho g)^{1 / 2}$, which is $2.7 \mathrm{~mm}$ for water. Thus gravity effects may be neglected and a spherical-cap assumption for the droplet holds [39]. For each test, a droplet is dispensed (using a carefully calibrated microsyringe) onto the test surface, and visualized using a goniometer imaging system (Model 290, ramé-hart) till it evaporates completely. The ambient temperature and relative humidity are maintained at $21 \pm 0.5^{\circ} \mathrm{C}$ and $36 \pm 2 \%$, respectively. The diffusivity of water vapor in air, as well as the saturated vapor concentration, is sensitive to temperature. The static contact angle of the droplet on the surface is measured using the goniometer. A cold light source used for backlighting ensures improved contrast without affecting the droplet evaporation rate. The images are recorded at intervals of 2 to $10 \mathrm{~s}$, with the finer resolution being used at the higher substrate temperatures. Each experiment is repeated at least four times to ensure repeatability of the results. The initial contact angle of the droplet on the smooth hydrophobic surface is $\sim 120^{\circ}$ and on the superhydrophobic surface is $\sim 160^{\circ}$. The corresponding values of contact angle hysteresis are $\sim 10^{\circ}$ and $\sim 1^{\circ}$, respectively.

\section{RESULTS AND DISCUSSION}

The experimental results for droplet evaporation on the hydrophobic and hierarchical superhydrophobic surfaces are discussed here in terms of the total time for evaporation, and the temporal variations of contact radius, contact angle, droplet volume, and the average instantaneous evaporation flux. Evaporation occurs primarily in a CCA mode on both substrates. The experimental results for the two surfaces are compared against each other and with predictions from the vapor-diffusion-only model. A simple model is presented that takes into account the temperature drop across the height of the droplet due to conduction through the droplet and the interface temperature dependence of the local saturated vapor concentration.

\section{A. Temporal variation of contact radius and contact angle}

Figure 2 shows the variation of droplet contact angle and nondimensional contact radius (nondimensionalized by the initial contact radius of the droplet) with respect to the time normalized by the total time of evaporation $\tau$ at three different substrate temperatures. The insets in Figs. 2(a) and 2(b) show the respective temporal evolution of droplet shape on the hydrophobic and superhydrophobic surfaces 


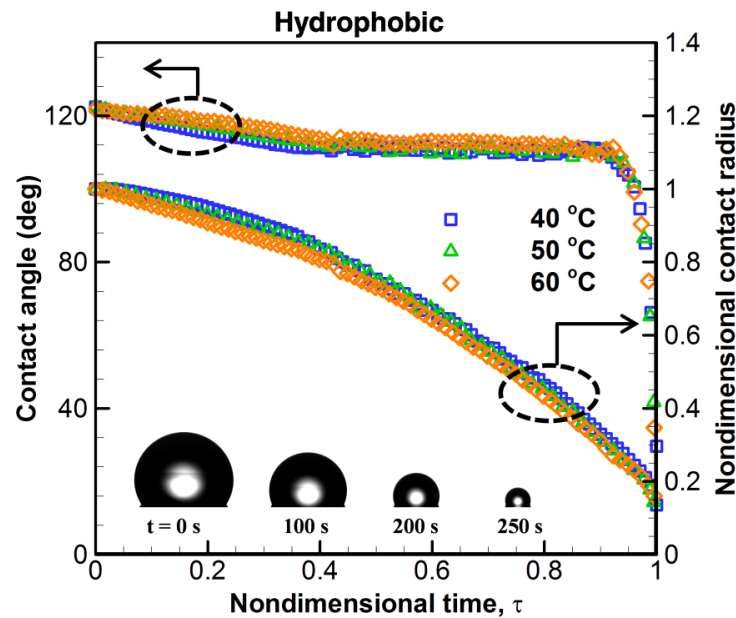

(a)

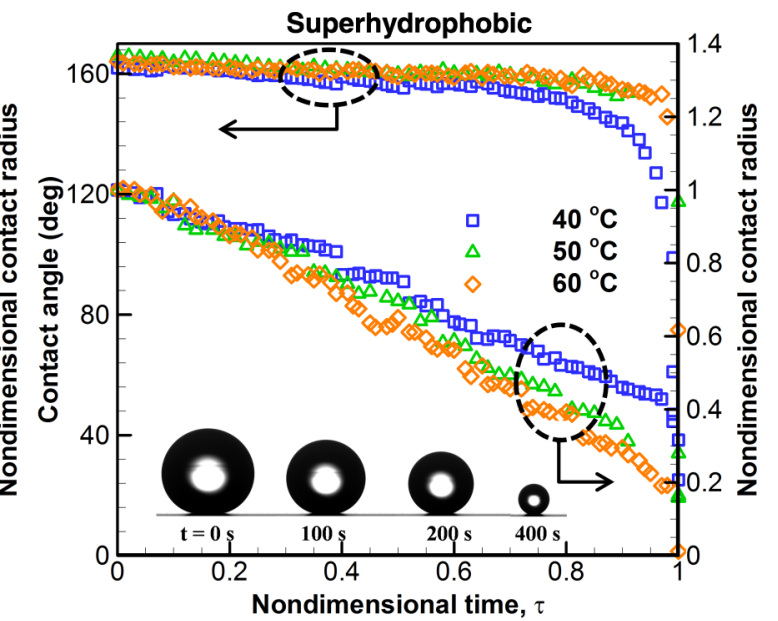

(b)

FIG. 2. (Color online) Variation of contact angle and nondimensional contact radius with respect to nondimensional time on the heated (a) hydrophobic surface and (b) superhydrophobic surface. The insets show the temporal variation of droplet shape corresponding to $T_{\text {sub }}=50{ }^{\circ} \mathrm{C}$.

corresponding to the substrate temperature $T_{\text {sub }}=50^{\circ} \mathrm{C}$. The variation with normalized time of the transient contact angles and nondimensional contact radii for different substrate temperatures is more or less similar. On the hydrophobic substrate, the contact radius of the droplet is observed to decrease continuously as the droplet evaporates as shown in Fig. 2(a). The droplet contact angle decreases from an initial contact angle to the receding contact angle value within the first $40 \%$ of the total time for evaporation. From then on, droplet evaporation occurs purely in a CCA mode till $\tau \sim 0.9$, followed by a mixed mode. This behavior is in contrast to droplet evaporation on unheated smooth hydrophobic surfaces [32] where a distinct CCR mode was observed and the contact radius remained fixed for the first $20 \%$ of the evaporation time; with substrate heating, the CCR mode is replaced by a mixed mode in which both the contact radius and contact angle decrease simultaneously. With the superhydrophobic surface, owing to the minimal contact angle hysteresis, the droplet evaporation occurs in a CCA mode for most of the evaporation period, as seen in Fig. 2(b). The average droplet contact angle during evaporation on the superhydrophobic surface remains at $\sim 160^{\circ}$. A stick-slip behavior is observed intermittently due to the surface roughness.

\section{B. Total time for evaporation}

It is important to assess the total time taken for the droplet to evaporate completely. In the presence of substrate heating, it is possible to reduce this time considerably as compared to an unheated case. Figure 3 shows the time taken for complete evaporation of a $3 \mu \mathrm{L}$ water droplet on the hydrophobic and superhydrophobic surfaces. Total time for evaporation corresponding to unheated substrates [32] is also included in the graph (substrate temperature of $21^{\circ} \mathrm{C}$ ). The times calculated from the vapor-diffusion model, Eq. (5), using the approximate value of the receding contact angles, i.e., $\theta=110^{\circ}$ for the hydrophobic substrate and $\theta=160^{\circ}$ for the superhydrophobic substrate, are also shown in the plot. The theoretical values of the total time of evaporation on the hydrophobic and superhydrophobic substrates overlap closely as shown in the plot. The experimental behavior supports the exponential relation between the total time and substrate temperature proposed by Girard et al. [37]. The dependence of total time for evaporation $t_{F}$ can be fitted with a power law, $t_{F}=a T_{\text {sub }}^{b}$ where $a=2510300, b=-2.34$ for the hydrophobic surface, and $a=767100, b=-1.91$, for the superhydrophobic surface. There is an excellent match between the measured total time for evaporation and that predicted by the vapor-diffusion model in the case of the smooth hydrophobic surface. Conversely, a considerable mismatch is observed when the surface is superhydrophobic. The vapor-diffusion model overpredicts the rate of evaporation on the superhydrophobic surface. This behavior is as opposed to droplet evaporation on a hydrophilic surface, where the isothermal diffusion-driven model was reported to underpredict the evaporation rate under heated conditions [34,36]. The time taken for complete evaporation of a droplet on the heated superhydrophobic surface is shown

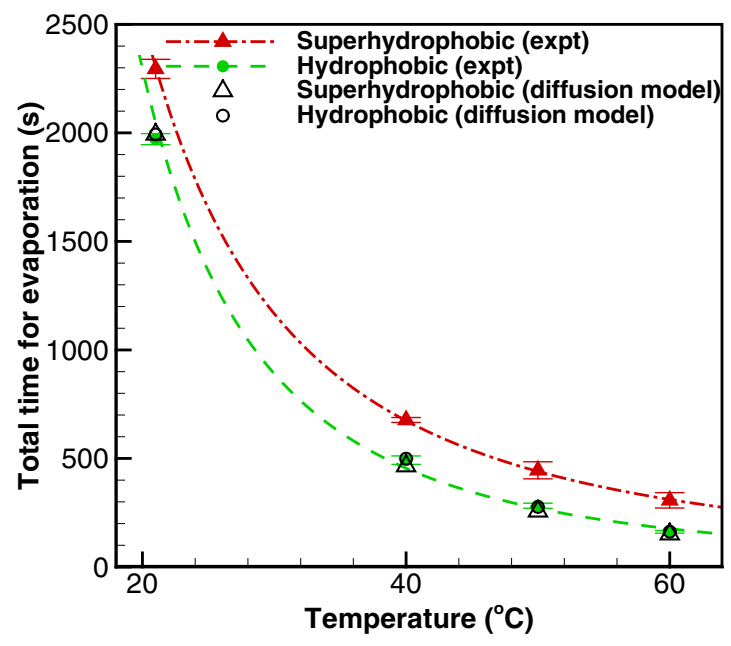

FIG. 3. (Color online) Total time for evaporation of droplets on the hydrophobic and superhydrophobic surfaces as a function of substrate temperature. The dashed lines represent a power fit to the experimental results. The hollow symbols represent the time for evaporation calculated from the vapor-diffusion model, Eq. (5). 
TABLE I. Total time for evaporation on the superhydrophobic substrate.

\begin{tabular}{lrcc}
\hline \hline $\begin{array}{l}\text { Substrate } \\
\text { temperature } \\
\left({ }^{\circ} \mathrm{C}\right)\end{array}$ & $\begin{array}{r}\text { Time taken for } \\
\text { evaporation (s): } \\
\text { Experimental }\end{array}$ & $\begin{array}{r}\text { Time taken for } \\
\text { evaporation (s): } \\
\text { Vapor-diffusion model }\end{array}$ & Deviation (\%) \\
\hline 40 & $677 \pm 11.4$ & 465 & 31.2 \\
50 & $445 \pm 39.3$ & 257 & 42.4 \\
60 & $307 \pm 35.5$ & 151 & 50.8 \\
\hline \hline
\end{tabular}

in Table I. The uncertainties shown represent the standard deviation of the different test runs. Table I shows the deviation between the measured and predicted times for total evaporation on the superhydrophobic substrate. The deviation is amplified with an increase in substrate temperature: from $31.2 \%$ at $40{ }^{\circ} \mathrm{C}$ to $50.8 \%$ at $60{ }^{\circ} \mathrm{C}$. At room temperature, without substrate heating, a $20 \%-25 \%$ deviation was reported for evaporation on a superhydrophobic surface in our earlier study [32].

\section{Variation of droplet volume}

The reduction in droplet volume over time during evaporation of the droplet is nonlinear for both hydrophobic and superhydrophobic surfaces (Fig. 4). This is similar to the behavior of droplet evaporation on hydrophobic and superhydrophobic surfaces without substrate heating [32]. Although the total time for droplet evaporation on the hydrophobic surface was found to be predicted very well by the vapor-diffusion model, the temporal variation of volume is not as well predicted [Fig. 4(a)]. This is in contrast with the droplet volume variation on an unheated hydrophobic surface, which was found to be predicted with remarkable accuracy by this model [32].

In the case of the superhydrophobic surface, the vapordiffusion model significantly overpredicts the experimental evaporation rate, and the percentage difference increases with an increase in substrate temperature [Fig. 4(b)]. The reason for the mismatch between the experimental results and the vapordiffusion model can be explained in terms of the competing

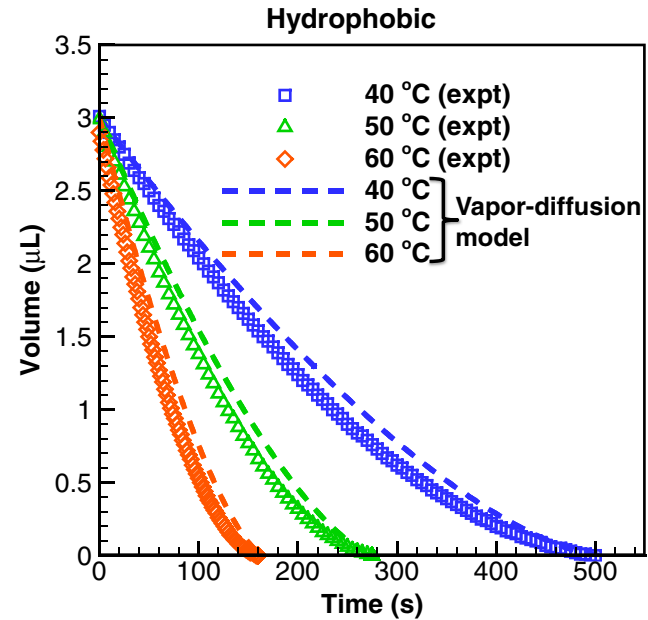

(a) effects of the buoyancy-induced convection in the vapor phase and the evaporative cooling along the interface [35], which will be described in detail in Sec. V.

\section{Variation of average evaporation flux}

The evaporation flux along the surface of an evaporating droplet is nonuniform except when the contact angle of the droplet is $90^{\circ}$ [7]. Based on the vapor-diffusion model, while the flux is diverging near the contact line of a droplet with contact angle $<90^{\circ}$, the flux is actually finite near the contact line when the contact angle is $>90^{\circ}$ [32]. Experimental determination of the local evaporation flux is challenging due to the difficulty in determination of the local vapor concentration as well as the temperature at the droplet interface. The area-averaged flux over the droplet surface is calculated using $J_{\text {avg }}=\left(\rho \frac{d V}{d t}\right) / A$ where $\rho, A$, and $V$ are the density, surface area, and volume of the droplet, respectively. Figures 5(a) and 5(b) show the variation of average evaporation flux with respect to the transient volume of the droplet for both surfaces. The average evaporation flux increases as the droplet evaporates on the hydrophobic and the superhydrophobic substrates; that is, the flux increases with reduction of the instantaneous droplet volume during evaporation. Towards the end of evaporation, corresponding to the period where droplet evaporation occurs in the mixed mode, there is a steep increase in the evaporation flux. For the same substrate temperature, the droplet evaporating on the hydrophobic surface has a higher evaporation flux as compared to the superhydrophobic surface, which can also be concluded from the lower time of evaporation in the case of evaporation on a hydrophobic surface.

\section{MODEL ACCOUNTING FOR CONDUCTION THROUGH THE DROPLET}

A model is developed here to demonstrate the influence of evaporative cooling on the rate of droplet evaporation on a heated substrate. The relative importance of the convective and the diffusive transport inside the droplet can be determined

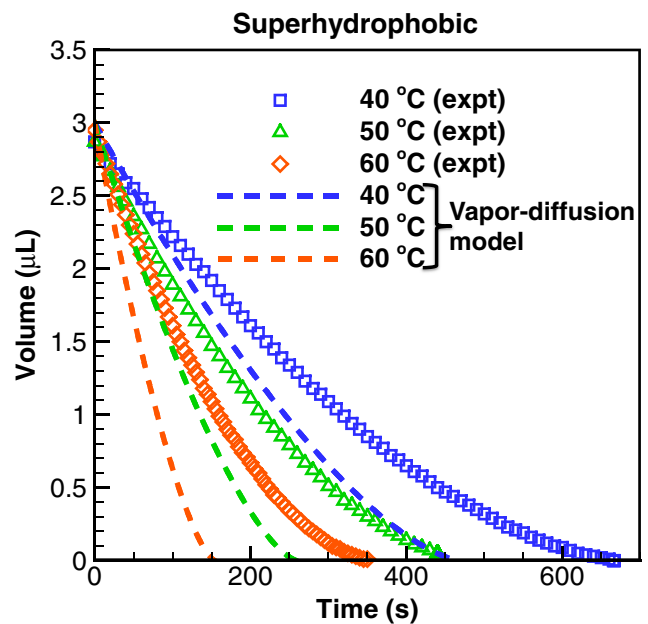

(b)

FIG. 4. (Color online) Temporal variation of droplet volume on the (a) hydrophobic and (b) superhydrophobic surfaces. The dashed lines represent the variation of droplet volume with respect to time as obtained from the vapor-diffusion model [Eq. (4)]. 


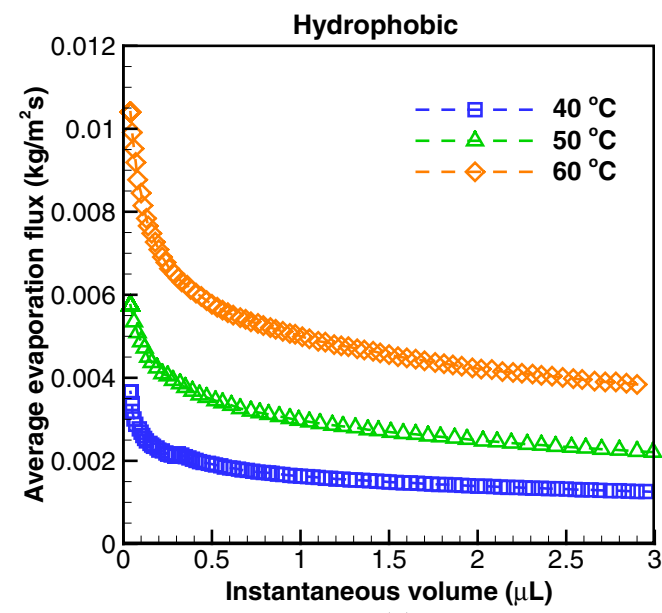

(a)

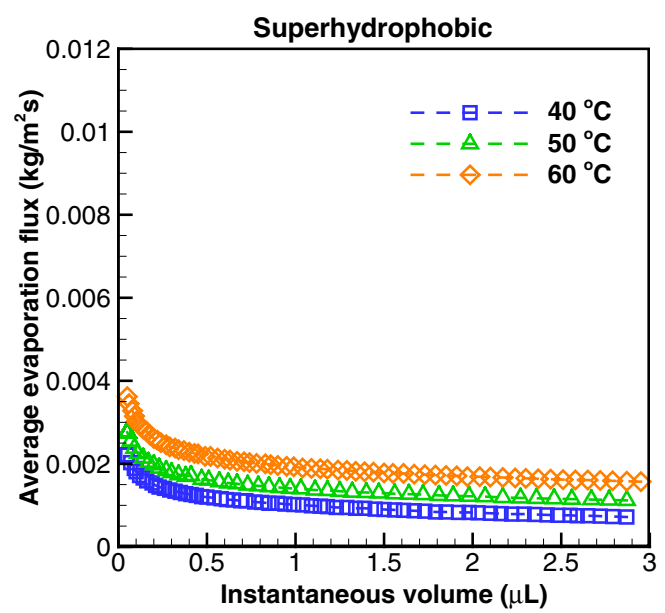

(b)

FIG. 5. (Color online) Average evaporation flux with respect to instantaneous volume for different substrate temperatures on the (a) smooth hydrophobic and (b) superhydrophobic substrates.

using the nondimensional Péclet number $(\mathrm{Pe}=U L / \alpha)$ where $U, L$, and $\alpha$ represent the characteristic velocity, length scale, and thermal diffusivity of the droplet, respectively. Using a characteristic velocity of tens of microns per second $[3,40]$, and the droplet height as the characteristic length scale, the Péclet number for a $3 \mu \mathrm{L}$ droplet is calculated to be less than 1 , signifying the dominance of the diffusive transport inside the liquid droplet. Similar conclusions regarding the minimal contribution of the convection inside the droplet on the net evaporation rate were made by Pan et al. [35] using a full-scale numerical model. The model developed in the present work accounts for thermal diffusion through the liquid droplet and vapor diffusion through the surrounding gas, while convection in the gas and liquid domains is neglected. The influence of the convection in the vapor domain can be analyzed based on the comparison between this model and the experimental results. Heat is conducted from the heated substrate through the droplet and utilized for phase change at the liquid-vapor interface. The substrate is held at a uniform constant temperature. The ambient temperature and humidity are taken as $21^{\circ} \mathrm{C}$ and $36 \%$ to match the experimental conditions. One-dimensional conduction is assumed along the vertical axis. The droplet volume is discretized into disks parallel to the droplet contact area, as shown in Fig. 6. The one-dimensional heat conduction model implies that the temperature laterally over each control

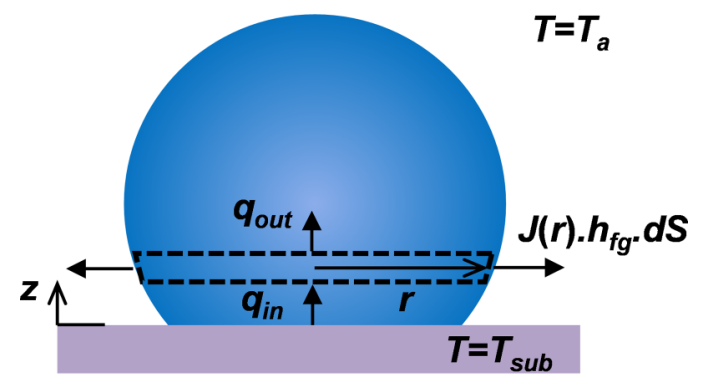

FIG. 6. (Color online) Control volume approach to determining the axial droplet interface temperature subject to evaporative cooling. volume remains uniform and the surface temperature variation occurs only along the vertical axis of the droplet. The energy balance over each control volume can be written as

$$
-\left.k A_{b} \frac{d T}{d x}\right|_{b}=-\left.k A_{t} \frac{d T}{d x}\right|_{t}+J(r) h_{f g} d S,
$$

where $k$ is the thermal conductivity of the liquid droplet $(k=$ $0.6 \mathrm{~W} / \mathrm{mK}$ for water), $A_{b}$ and $A_{t}$ are respectively the bottom and top face areas of the control volume, $h_{f g}$ is the latent heat of vaporization, and $d S$ corresponds to the surface area of the control volume representing the liquid-air interface. The evaporation flux $J(r)$ in Eq. (6) is initialized as the theoretical flux obtained from the vapor-diffusion model using Eq. (1) and evaluated at the substrate temperature. The thermophysical properties are evaluated at the interface temperature (initialized as the substrate temperature). The saturated vapor concentration at the droplet interface is coupled with the saturation pressure and hence the local interface temperature at the droplet. The saturation pressure $p_{\text {sat }}\left(T_{l v}\right)$ is calculated using the Clausius-Clapeyron equation:

$$
\begin{aligned}
p_{\text {sat }}\left(T_{l v}\right) & =p_{\text {sat_ref }} \exp \left[\frac{M h_{f g}}{R}\left(\frac{1}{T_{\text {sat_ref }}}-\frac{1}{T_{l v}}\right)\right], \\
\left.C_{v}\right|_{l v} & =\frac{p_{\text {sat }}\left(T_{l v}\right)}{R T_{l v}}, \\
h_{f g} & =2.7554 \times 10^{6}-3.46 T^{2} .
\end{aligned}
$$

The energy equation (6) is solved to obtain temperatures using the finite volume method in MATLAB using a forwarddifference scheme. The updated local temperatures are used to determine the evaporation flux and the thermophysical parameters $\left(P_{\text {sat }}, C_{v}, h_{f g}\right)$ in subsequent iterations. The energy equation is solved and iterated upon until convergence and the resultant surface temperature profile is obtained along the vertical axis of the droplet.

The interfacial temperatures of a $3 \mu \mathrm{L}$ droplet obtained by solution of Eq. (6), with contact angles of $110^{\circ}$ and $160^{\circ}$, are plotted in Figs. 7(a) and 7(b), respectively. The magnitude of the evaporative cooling is assessed based on $\Delta T$, the 


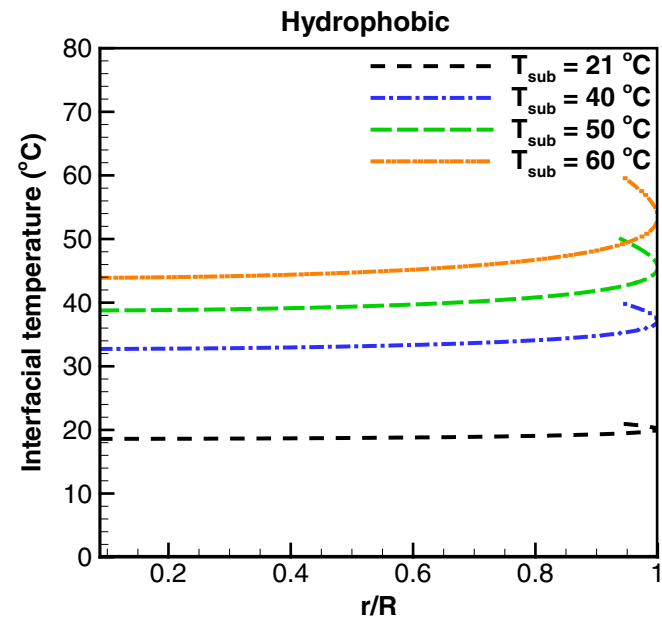

(a)

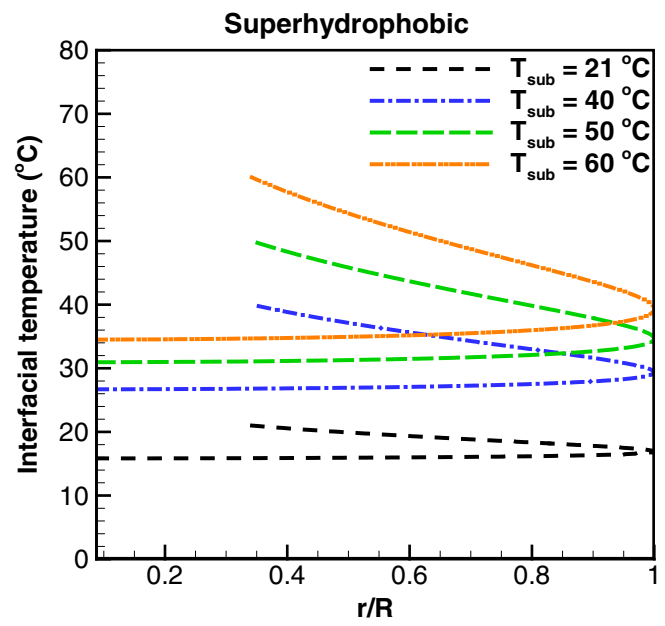

(b)

FIG. 7. (Color online) Interfacial temperature of a droplet of $3 \mu \mathrm{L}$ volume at different substrate temperatures corresponding to the (a) hydrophobic $\left(\theta=110^{\circ}\right)$ and (b) superhydrophobic substrates $\left(\theta=160^{\circ}\right)$. The radial location $r$ along the interface is normalized by the droplet radius $(R)$.

difference between the substrate temperature and the minimum temperature which occurs at the top of the droplet interface, as shown in Table II. The magnitude of interfacial cooling increases with an increase in substrate temperature for both the contact angles considered (Table II). For droplet evaporation in the CCA mode, the total time of evaporation is related to the inverse of the rate of evaporation, as seen from Eqs. (3) and (5). The ratio of the measured evaporation rate $d m /\left.d t\right|_{\text {expt }}$ and that given by the vapor-diffusion-only model $d m /\left.d t\right|_{\text {diff }}$ is hence obtained as the ratio of the total time for evaporation from Eq. (5) and from the measured values in the form of $\left(d m /\left.d t\right|_{\text {expt }}\right) /\left(d m /\left.d t\right|_{\text {diff }}\right) \sim\left(t_{F}\right)_{\text {diff }} /\left(t_{F}\right)_{\text {expt }}$. The evaporation rate increases with substrate heating, resulting in an increased rate of cooling, and sustains a larger temperature differential across the droplet.

The effect of evaporative cooling on the interface temperature is much larger in the case of the superhydrophobic surface compared to the smooth hydrophobic surface for the substrate temperatures considered (Table II). This can be explained in terms of the higher height-to-contact-radius aspect ratio of a droplet on the superhydrophobic surface than on the hydrophobic surface, $h / R_{c}=5.67\left(\mathrm{CA}=160^{\circ}\right)$ versus $h / R_{c}=1.43$ $\left(\mathrm{CA}=110^{\circ}\right)$. The longer conduction path $(h)$ and a significantly lower conduction base area $\left(A_{c}=\pi R_{c}^{2}\right)$ in the case of the superhydrophobic surface as compared to the hydrophobic surface result in a larger temperature differential in the former. In contrast, for a hydrophilic surface, the low contact angle of the droplet implies a significantly lower $h / R_{c}$ aspect ratio. The temperature drop across a droplet on hydrophilic surfaces is thus minimal, as reported by Girard et al. [37].

Despite the considerable temperature drop predicted for the hydrophobic substrate, the total time for evaporation recorded experimentally shows excellent agreement with the vapor-diffusion-only model (Table II). A similar observation was made by Pan et al. [35], who reported that under unheated substrate conditions with a droplet contact angle of $110^{\circ}$, the suppression of evaporation caused by the evaporative cooling effect is compensated by the convective flow in the air which tends to enhance the rate of evaporation. The agreement between the current experimental results and the vapordiffusion-only model even at elevated temperature indicates that the buoyancy-driven convection balances the evaporative cooling effect even as the interface cooling increases with an increase in the substrate temperature. This implies that the evaporation enhancement effect of buoyancy-induced convection would be amplified as the substrate temperature is increased. Conversely, for the superhydrophobic surface, there is a considerable difference between the measured and predicted time of evaporation. This indicates that the enhancement of evaporation rate due to convection in the air and liquid domains is not sufficient to compensate for evaporation suppression due to the lowered interface temperature. For hydrophilic surfaces, on the other hand, the vapor-diffusion model has been shown to underpredict the rate of evaporation

TABLE II. Interfacial temperature of droplet subject to evaporative cooling.

\begin{tabular}{|c|c|c|c|c|}
\hline \multirow[b]{2}{*}{$T_{\text {sub }}\left({ }^{\circ} \mathrm{C}\right)$} & \multicolumn{2}{|c|}{ Hydrophobic substrate $\left(\theta=110^{\circ}\right)$} & \multicolumn{2}{|c|}{ Superhydrophobic substrate $\left(\theta=160^{\circ}\right)$} \\
\hline & $\Delta T\left({ }^{\circ} \mathrm{C}\right)$ & Evaporation time ratio $\left(t_{F}\right)_{\text {diff }} /\left(t_{F}\right)_{\text {expt }}$ & $\Delta T\left({ }^{\circ} \mathrm{C}\right)$ & Evaporation time ratio $\left(t_{F}\right)_{\mathrm{diff}} /\left(t_{F}\right)_{\text {expt }}$ \\
\hline 21 & 2.41 & 1.011 & 5.17 & 0.770 \\
\hline 40 & 7.28 & 1.003 & 13.3 & 0.687 \\
\hline 50 & 11.22 & 0.984 & 19.05 & 0.576 \\
\hline 60 & 16.09 & 0.970 & 25.49 & 0.492 \\
\hline
\end{tabular}


when subject to substrate heating $[33,35]$. This is because the suppression of the evaporation rate by lowering the interface temperature is negligible in the case of the hydrophilic surface, and the effect of buoyant convection-induced enhancement of the evaporation rate is dominant.

\section{CONCLUSION}

The influence of substrate temperature on the evaporation characteristics of droplets on hydrophobic and superhydrophobic surfaces is experimentally investigated. The experimental results are compared with a vapor-diffusion model which assumes the droplet temperature to be equal to the temperature of the substrate. With the hydrophobic substrate, there is good agreement between the measurements and model predictions for the time taken for total evaporation. In contrast, the evaporation results on the superhydrophobic surface deviate significantly from the vapor-diffusion model, both in terms of the total time for evaporation and the variation of transient geometric parameters of the droplet. The neglect of the evaporative cooling effect at the droplet interface, the temperature gradient established along the height of the droplet, and the resultant nonuniform interface temperature, cause a mismatch between the experimental results and the vapor-diffusion model; this disagreement is amplified with an increase in the substrate temperature. A simple model is solved to determine the nonuniform interface temperature which is lower than the substrate temperature. This model highlights the importance of evaporative cooling on reducing the resultant rate of evaporation of the droplet, especially in the case of superhydrophobic surfaces.

\section{ACKNOWLEDGMENT}

Funding from the National Science Foundation for this work as a Fundamental Research Supplement to the Cooling Technologies Research Center at Purdue University is gratefully acknowledged.
[1] P. Calvert, Chem. Mater. 13, 3299 (2001).

[2] N. Kumari and S. V. Garimella, Int. J. Heat Mass Transf. 54, 4037 (2011).

[3] H. K. Dhavaleswarapu, C. P. Migliaccio, S. V. Garimella, and J. Y. Murthy, Langmuir 26, 880 (2010).

[4] Q. Li, Y. T. Zhu, I. A. Kinloch, and A. H. Windle, J. Phys. Chem. B 110, 13926 (2006).

[5] S. T. Chang and O. D. Velev, Langmuir 22, 1459 (2006).

[6] R. D. Deegan, O. Bakajin, T. F. Dupont, G. Huber, S. R. Nagel, and T. A. Witten, Phys. Rev. E 62, 756 (2000).

[7] Y. O. Popov, Phys. Rev. E 71, 036313 (2005).

[8] A. Ebrahimi, P. Dak, E. Salm, S. Dash, S. V. Garimella, R. Bashir, and M. A. Alam, Lab Chip 13, 4248 (2013).

[9] J. Jing, J. Reed, J. Huang, X. Hu, V. Clarke, J. Edington, D. Housman, T. S. Anantharaman, E. J. Huff, and B. Mishra, Proc. Natl. Acad. Sci. U.S.A. 95, 8046 (1998).

[10] A. Wu, L. Yu, Z. Li, H. Yang, and E. Wang, Anal. Biochem. 325, 293 (2004).

[11] K. S. Birdi and D. T. Vu, J. Adhes. Sci. Technol. 7, 485 (1993).

[12] S. A. Kulinich and M. Farzaneh, Appl. Surf. Sci. 255, 4056 (2009).

[13] N. Anantharaju, M. Panchagnula, and S. Neti, J. Colloid Interface Sci. 337, 176 (2009).

[14] R. G. Picknett and R. Bexon, J. Colloid Interface Sci. 61, 336 (1977).

[15] G. McHale, S. M. Rowan, M. I. Newton, and M. K. Banerjee, J. Phys. Chem. B 102, 1964 (1998).

[16] Y.-S. Yu, Z. Wang, and Y.-P. Zhao, J. Colloid Interface Sci. 365, 254 (2012).

[17] S. David, K. Sefiane, and L. Tadrist, Colloids Surf. A 298, 108 (2007).

[18] G. J. Dunn, S. K. Wilson, B. R. Duffy, S. David, and K. Sefiane, J. Fluid Mech. 623, 329 (2009).

[19] H. Hu and R. G. Larson, J. Phys. Chem. B 106, 1334 (2002).

[20] R. D. Deegan, O. Bakajin, T. F. Dupont, G. Huber, S. R. Nagel, and T. A. Witten, Nature (London) 389, 827 (1997).

[21] J. Ollesch, S. L. Drees, H. M. Heise, T. Behrens, T. Brüning, and K. Gerwert, Analyst 138, 4092 (2013).
[22] E. Tekin, P. J. Smith, and U. S. Schubert, Soft Matter 4, 703 (2008).

[23] Á. G. Marín, H. Gelderblom, A. Susarrey-Arce, A. van Houselt, L. Lefferts, J. G. E. Gardeniers, D. Lohse, and J. H. Snoeijer, Proc. Natl. Acad. Sci. U.S.A. 109, 16455 (2012).

[24] G. McHale, S. Aqil, N. J. Shirtcliffe, M. I. Newton, and H. Y. Erbil, Langmuir 21, 11053 (2005).

[25] S. Dash, N. Kumari, and S. V. Garimella, J. Micromech. Microeng. 21, 105012 (2011).

[26] H. Y. Erbil, G. McHale, and M. I. Newton, Langmuir 18, 2636 (2002).

[27] M. E. R. Shanahan, K. Sefiane, and J. R. Moffat, Langmuir 27, 4572 (2011).

[28] X. Zhang, S. Tan, N. Zhao, X. Guo, X. Zhang, Y. Zhang, and J. Xu, ChemPhysChem 7, 2067 (2006).

[29] H. Gelderblom, A. G. Marin, H. Nair, A. van Houselt, L. Lefferts, J. H. Snoeijer, and D. Lohse, Phys. Rev. E 83, 026306 (2011).

[30] F. D. Angelis, F. Gentile, F. Mecarini, G. Das, M. Moretti, P. Candeloro, M. L. Coluccio, G. Cojoc, A. Accardo, C. Liberale, R. P. Zaccaria, G. Perozziello, L. Tirinato, A. Toma, G. Cuda, R. Cingolani, and E. D. Fabrizio, Nat. Photon. 5, 682 (2011).

[31] P. Brunet, Soft Matter 8, 11294 (2012).

[32] S. Dash and S. V. Garimella, Langmuir 29, 10785 (2013).

[33] T. A. Nguyen, A. V. Nguyen, M. A. Hampton, Z. P. Xu, L. Huang, and V. Rudolph, Chem. Eng. Sci. 69, 522 (2011).

[34] B. Sobac and D. Brutin, Phys. Rev. E 86, 021602 (2012).

[35] Z. Pan, S. Dash, J. A. Weibel, and S. V. Garimella, Langmuir 29, 15831 (2013).

[36] F. Carle, B. Sobac, and D. Brutin, Appl. Phys. Lett. 102, 061603 (2013).

[37] F. Girard, M. Antoni, S. Faure, and A. Steinchen, Colloids Surf. A 323, 36 (2008).

[38] MATLAB, Reference Manual, The Mathworks Inc., Natick, MA, 2007.

[39] D. Quere, Rep. Prog. Phys. 68, 2495 (2005).

[40] Á. G. Marín, H. Gelderblom, D. Lohse, and J. H. Snoeijer, Phys. Rev. Lett. 107, 085502 (2011). 